\title{
Pituitary tumours and pregnancy
}

\author{
Michael Powell
}

Received: 23 May 2011 / Accepted: 24 May 2011 / Published online: 10 June 2011

(C) Springer-Verlag 2011

The pregnant neurosurgical emergency patient is fortunately extremely rare. Management decisions in strictly neurosurgical terms are relatively straightforward, but surgical decisions will often be compromised by the needs of the developing baby and particularly the risks to it. The main problems are in the restrictions in anaesthesia and imaging, these specialities having far more experience in dealing with a pregnant patient than our own. The fear of damaging the baby and the consequent medico-legal litigation risk poses significant restrictions on what these specialties are prepared to do.

Ultimately it comes down to clinical urgency assessed against risk to the baby.

A clear algorithm for neurosurgical management has been already published by $\mathrm{Ng}$ and Kitchen [1], and the series, in this edition from Israel [2], in effect backs this management plan up. However, these authors conclude that known tumours, in their series glial and meningiomas, are likely to create problems at some stage in the course of the pregnancy. This may well be the case; however, the series is, as the authors have noted, perhaps unusual in that there were no patients managed with pituitary adenomas.

Pituitary neurosurgeons involved in the multi-disciplinary management of adenoams regularly see pituitary women patients who wish to concieve, or who do so and then have to be managed throughout the pregnancy with their tumour.

The most common are the prolactinoma patients who are usually managed simply medically on the dopamine agonist, cabergoline. This drug is currently not licensed for use in pregnancy. Most endocrinologists will transfer the

\section{Powell $(\bowtie)$}

The National Hospital for Neurology \& Neurosurgery,

Queen Sq,

London WC1N 3BG, UK

e-mail: michael.powell@uclh.nhs.uk patient to less effective bromocryptine, which is licensed. As will be well known, its side effect profile, although similar, is more strongly expressed than cabergoline. Thus, with the nausea often associated with pregnancy, many patients stop dopamine agonist therapy altogether, and thus run the risk of the prolactinoma enlarging.

In microprolactinomas this is of no real consequence, but not all endocrinologists understand this and certainly few obstetricians. This often will lead to complex management discussions with those specialties. Since lactotroph hypertrophy is also a natural consequence of pregnancy, the gland enlarges and with it the serum prolactin levels increase to at least tenfold over normal control values. Reassurance is all that is required.

For macroprolactinoma patients the story is rather different. The tumour may well re-grow off medication and compromise vision. If they are either unresponsive or intolerant to bromocryptine, then either the patient has to be replaced on cabergoline with the knowledge of the potential risks (despite the manufacturers concerns, actually rather small), or, if the tumour rapidly increases, then de-bulking surgery may well be required. Significant visual field change for the worse is the triggering event for surgery

The story is similar for non-secreting macro-adenomas. There is no clear evidence that the tumours themselves increase during pregnancy, but anecdotally, most large series can report an occasional tumour that does so. In this author's personal series, this has occurred in two silent corticotroph adenomas. In any case, as mentioned above, the normal lactotroph cells hypertrophy and so any tumour or residual tumour mass will apparently increase as the normal part of the gland swells. Some endocrinologists will then try bromocryptine or (even cabergoline which ought to be safe in the latter stages of pregnancy when this is most likely to occur) in an attempt to shrink this hypertrophy. 
Apart from vigilance with regular, perhaps monthly, eye field testing, there is not much else that can be recommended, as frequent imaging, either computed tomography or magnetic resonance imaging, (particularly in the first trimester) is usually discouraged by imaging departments. In any event, surgery may be required, although it is not that frequently so. Trans-sphenoidal surgery in a pregnant women is no more difficult than normal from the neurosurgical point of view, although fluoroscopy may be slightly restricted, if used. The option of early delivery prior to a neurosurgical procedure is also an option.

Two other events may occur in the later stages of pregnancy relating to pituitary disease. Apoplexy is occasionally recorded. The recent guidelines for management from the UK Pituitary Apoplexy Multi-Disciplinary Steering Group [3] can be followed here, and surgery only needed if visual fields are significantly compromised.

The other event which particularly occurs in the peri-partum period is lymphocytic hypophisitis of the adenopophysis, although it occasionally affects non-parous women and, even in a few reported cases, men. Since it is usually associated with dramatic hypopituitarism with an unusually low prolactin for the peri-partum period and even including diabetes insipidus, the diagnosis should be straightforward. The imaging is also well described, often with a tail of brightly enhancing thickening up the stalk. Surgery, even when visual fields are significantly reduced, should not be required as these patients respond quickly and well to high-dose steroids for a relatively short period of time, both in vision and pituitary function terms. Unfortunately however, the reduction in vision is often used to mandate a surgical decision. The gland in this setting is very tough and since the surgeon is removing what would at some stage return to be a normal functioning gland, then the patient's normal pituitary function will never recover.

Conflicts of interest. None.

\section{References}

1. Ng J, Kitchen N (2008) Neurosurgery and pregnancy. J Neurol Neurosurg Psychiatry 79(7):745-52

2. Nossek E, Ekstein M, Rimon E, Kupferminc MJ, Ram Z (2011) Neurosurgery and pregnancy. Acta Neurochir (Wien) (in press)

3. Rajasekaran S, Vanderpump M, Baldeweg S, Drake W, Reddy N, Markey A, Plant G, Powell M, Sinha S, Wass J (2011) UK guidelines for management of pituiatary apoplexy. Clin Endoc 74 (1):9-20 